\title{
The screening potentials of ion-dipole system adsorbed in ion-dipolar disordered matrices
}

\author{
M.F.Holovko, Z.V.Polishchuk \\ Institute for Condensed Matter Physics \\ of the National Academy of Sciences of Ukraine, \\ 1 Svientsitskii Str., 290011 Lviv, Ukraine
}

Received September 4, 1998

\begin{abstract}
The replica Ornstein-Zernike equations ion-molecular fluid adsorbed in a disordered ion-molecular matrix were applied. The explicit expressions for the potentials screening by the point particles are obtained. The analysis of the screening potentials for ion-dipolar case are presented. It is shown that fluid-fluid interionic potentials include connected and blocked parts.
\end{abstract}

Key words: replica Ornstein-Zernike equations, screening potentials, porous media, ion-molecular system

PACS: $61.20,05.20$

The effects of quenched disorder on the fluid properties have received increased attention in recent years. In theoretical studies the matrix-fluid system is considered as a special binary mixture in which the matrix is treated as a rigid set of obstacles,obtained by quenching an equilibrium configuration of particles (usually noted as species "0") with the fluid molecules (noted as species "1") at equilibrium in the presence of quenched particles. Resulting from the applying of the replica trick the description of the original matrix-fluid system was replaced by a multicomponent equilibrium $(s+1)$ component mixture for which it was possible to use the standard methods of liquid-state theory (the integral equations, cluster expansions ets.). The specific feature of such $(s+1)$-component mixture is that a pair of fluid particles from different replicas has no interaction and at the end of calculation it takes the limit $s \rightarrow 0$, assuming that there is no problem in performing the analytical continuation for noninteger values of $s$.

In this way Given and Stell [1-2] recently proposed a set of coupled integral equations, the so-called replica Ornstein-Zernike (ROZ) equations, relating the total pair correlation functions $h_{\alpha \beta}(r)$ of fluid-matrix mixture to the corresponding direct 
correlation functions $c_{\alpha \beta}(r)$. Using the Fourier-transform

$$
\hat{f}(k)=4 \pi \int_{0}^{\infty} r^{2} \mathrm{~d} r \frac{\sin k r}{k r} f(r)
$$

the formal solution of these ROZ equations can be represented in the following form

$$
\begin{aligned}
\hat{h}_{00}(k) & =\frac{\hat{c}_{00}(k)}{1-\rho_{0} \hat{c}_{00}(k)}, \\
\hat{h}_{01}(k) & =\frac{\hat{c}_{01}(k)}{\left[1-\rho_{0} \hat{c}_{00}(k)\right]\left[1-\rho_{1} \hat{c}_{11}^{\mathrm{c}}(k)\right]}, \\
\hat{h}_{11}^{\mathrm{c}}(k) & =\frac{\hat{c}_{11}^{\mathrm{c}}(k)}{1-\rho_{1} \hat{c}_{11}^{\mathrm{c}}(k)}, \\
\hat{h}_{12}(k) & =\frac{\hat{c}_{12}(k)}{\left[1-\rho_{1} \hat{c}_{11}^{\mathrm{c}}(k)\right]^{2}}+\frac{\rho_{0} \hat{c}_{01}(k) \hat{c}_{10}(k)}{\left[1-\rho_{0} \hat{c}_{00}(k)\right]\left[1-\rho_{1} \hat{c}_{11}^{\mathrm{c}}(k)\right]^{2}},
\end{aligned}
$$

where due to the symmetry $h_{01}(r)=h_{10}(r), c_{01}(r)=c_{10}(r), \rho_{0}$ and $\rho_{1}$ are average density of matrix and fluid particles correspondingly. The fluid-fluid pair correlation functions are decomposed into two parts

$$
h_{11}(r)=h_{11}^{\mathrm{c}}(r)+h_{12}(r), \quad c_{11}(r)=c_{11}^{\mathrm{c}}(r)+c_{12}(r),
$$

where $h_{11}^{\mathrm{c}}(r)$ and $c_{11}^{\mathrm{c}}(r)$ are the connected parts; $h_{12}(r)=h_{11}^{\mathrm{b}}(r)$ and $c_{12}(r)=c_{11}^{\mathrm{b}}(r)$ are the correlation functions of two fluid molecules from different replica copies, so-called blocked part of the fluid-fluid pair correlation functions.

For this time the application of ROZ equations was restricted the description of simple fluids in random and quenched matrices [3-6].Only recently a study of associating fluids [6-7] and ionic fluids [8-9] in porous media using ROZ equations was initiated. In particular, in [8] the ROZ equations were applied for the investigation of the screening potentials for the proposed in [10] model of ionic fluids adsorbed in an electroneutral totally disordered matrix of ion. In this model both ionic subsystems were presented as point charges interacting via Coulomb interactions. The important influence of the blocking effects of the quenched matrix on the screening between the fluid ions was shown. The obtained screening potentials can be used to build the cluster expansions of pair correlation functions and free energy of the system [11-12] or to develop the renormalization scheme for the long-range terms in ionion correlations for the numerical solution ROZ equations [9]. However the model considered and the results obtained should be, as usual, [11-12] revised in terms of more civilized ion-molecular models which take into account explicitly the solvent molecules and dielectric peculiarities of the matrix.

Investigating the screening potentials of such ion-molecular fluid-matrix model is the aim of this article. We consider a general case of two electroneutral threecomponent ion-molecular subsystems. The first one represents the matrix and the other one is fluid. As regards the usual ion-molecular systems [11-12] the ions have 
the charges $e z_{e}^{\alpha}$ and the molecules characterized by the generalized charges $Q_{\mathrm{s}}^{\alpha}(\nabla)$, where index $\alpha=0$ or 1 and denotes the quenched and fluid component respectively. The index $a$ denotes the ions and the index $s$ denotes the molecules. For compactness we will also use the indices $x, y$, which include ions and molecules

$$
Q_{x}^{\alpha}(\nabla)= \begin{cases}e z_{a}^{\alpha}, & x=a \\ Q_{\mathrm{s}}^{\alpha}(\nabla), & x=s\end{cases}
$$

Thus, the electrostatic interactions between the particles in the model considered can be represented in the following form

$$
\Phi_{x y}^{\alpha \beta}(r)=Q_{x}^{\alpha}(\nabla) Q_{y}^{\beta}(-\nabla) \frac{1}{R} .
$$

For simplicity, in this case we consider: the molecules with point dipole moment $\vec{p}_{\mathrm{s}}$ [11-12] for which

$$
Q_{\mathrm{s}}^{\alpha}(\nabla)=\left(p_{\mathrm{s}}^{\alpha} \nabla\right)
$$

In order to obtain the expressions for the screening potentials by point particles we should put [11-12]

$$
C_{x y}^{\alpha \beta}(r)=-\frac{1}{k_{\mathrm{B}} T} \Phi_{x y}^{\alpha \beta}(r), \quad h_{x y}^{\alpha \beta}(r)=G_{x y}^{\alpha \beta}(r),
$$

where $G_{x y}^{\alpha \beta}(r)$ are the screening potentials, $\Phi_{x y}^{\alpha \beta}(r)$ are given by equation (8) and $\Phi_{x y}^{12}(r)=0$ since the particles from different replicas do not interact.

By expanding the Coulomb potential $1 / r$ in a Fourier series the electrostatic potentials $\Phi_{x y}^{\alpha \beta}(r)$ can be represented in the following form

$$
\Phi_{x y}^{\alpha \beta}(r)=\sum_{k} \frac{1}{V} \frac{4 \pi}{k^{2}} \hat{Q}_{x}^{\alpha}(k) Q_{y}^{\beta}(-k) e^{\mathrm{i} \bar{k} \bar{r}},
$$

where $\hat{Q}_{x}^{\alpha}(k)=e z_{a}^{\alpha}$ for $x=a$ and $\hat{Q}_{x}^{\alpha}(k)=\hat{Q}_{\mathrm{s}}(k)=\mathrm{i}\left(p_{\mathrm{s}}^{\alpha} k\right)$ for $x=s$.

Now according to expressions (2)-(5) and in assumption (10) the Fourier transform of the screening potentials can be explicitly represented as follows:

$$
\begin{aligned}
\hat{G}_{x y}^{00}(k) & =-\frac{1}{k_{\mathrm{B}} T} \frac{\hat{Q}_{x}^{0}(k) \hat{Q}_{y}^{0}(-k)}{\left(\varepsilon_{0}(k)+\frac{\kappa_{0}^{2}}{k^{2}}\right) k^{2}} \\
\hat{G}_{x y}^{01}(k) & =-\frac{1}{k_{\mathrm{B}} T} \frac{Q_{x}^{0}(k) Q_{y}^{0}(-k)}{k^{2}\left[\varepsilon_{0}(k)+\frac{\kappa_{0}^{2}}{k^{2}}\right]\left[\varepsilon_{1}(k)+\frac{\kappa_{1}^{2}}{k^{2}}\right]} \\
\hat{G}_{x y}^{11}(k) & =\hat{G}_{x y}^{\mathrm{c}, 11}(k)+\hat{G}_{x y}^{12}(k), \\
\hat{G}_{x y}^{\mathrm{c}, 11}(k) & =-\frac{1}{k_{\mathrm{B}} T} \frac{\hat{Q}_{x}(k) Q_{y}^{1}(-k)}{k^{2}\left(\varepsilon_{1}(k)+\frac{\kappa_{1}^{2}}{k^{2}}\right)}
\end{aligned}
$$




$$
\begin{aligned}
\hat{G}_{x y}^{12}(k) & =\frac{1}{k_{\mathrm{B}} T} Q_{x}^{1}(k) Q_{y}^{1}(-k) \frac{1}{k^{2}} \\
& \times\left[\frac{\varepsilon_{0}(k)-1}{\varepsilon_{0}(k)} \frac{1}{\left(\varepsilon_{1}(k)+\frac{\kappa_{1}^{2}}{k^{2}}\right)^{2}}+\frac{\kappa_{0}^{2}}{\varepsilon_{0}(k) k^{2}} \frac{1}{\left(\frac{\kappa_{0}^{2}}{k^{2}}+\varepsilon_{0}(k)\right)\left(\varepsilon_{1}(k)+\frac{\kappa_{1}^{2}}{k^{2}}\right)^{2}}\right]
\end{aligned}
$$

where $\kappa_{0}=\left[4 \pi \frac{1}{k_{\mathrm{B}} T} \sum a \rho_{a}^{0} e^{2}\left(z_{a}^{0}\right)^{2}\right]^{\frac{1}{2}}, \kappa_{1}=\left[4 \pi \frac{1}{k_{\mathrm{B}} T} \sum a \rho_{a}^{1} e^{2}\left(z_{a}^{1}\right)^{2}\right]^{\frac{1}{2}}$ is the reciprocal Debye-Huckel radius of screening for the matrix and fluid ionic subsystem, respectively. As usual [11-12], the molecular screening introduces the static dielectric functions for the matrix and fluid subsystems, respectively

$$
\begin{aligned}
& \varepsilon_{0}(k)=1+4 \pi \frac{1}{k_{\mathrm{B}} T} \rho_{\mathrm{s}}^{0} \int \mathrm{d} \Omega_{\mathrm{s}}^{0} \frac{1}{k^{2}} Q_{\mathrm{s}}^{0}(k) Q_{\mathrm{s}}^{0}(-k)=\varepsilon_{0}=1+3 y_{0}, \\
& \varepsilon_{1}(k)=1+4 \pi \frac{1}{k_{\mathrm{B}} T} \rho_{\mathrm{s}}^{1} \int \mathrm{d} \Omega_{\mathrm{s}}^{1} \frac{1}{k^{2}} Q_{\mathrm{s}}^{1}(k) Q_{\mathrm{s}}^{1}(-k)=\varepsilon_{1}=1+3 y_{1},
\end{aligned}
$$

which have the meaning of the dielectic constant of matrix and fluid subsystem respectively, $y_{0}=\frac{4}{3} \pi \frac{1}{k_{\mathrm{B}} T} \rho_{\mathrm{s}}^{0}\left(p_{\mathrm{s}}^{0}\right)^{2}, y_{1}=\frac{4}{3} \pi \frac{1}{k_{\mathrm{B}} T} \rho_{\mathrm{s}}^{1}\left(p_{\mathrm{s}}^{1}\right)^{2}$.

The expressions (12)-(16) are the main result of this article. For the ion-dipolar case $\varepsilon_{0}(k)=\varepsilon_{0}$ and $\varepsilon_{1}(k)=\varepsilon_{1}$ the screening potentials in coordinate space have the following form

$$
\begin{aligned}
G_{x y}^{00}(R) & =-\frac{1}{k_{\mathrm{B}} T} Q_{x}^{0}(\nabla) Q_{y}^{0}(-\nabla) \frac{1}{\varepsilon_{0} r} \exp \left(-\frac{\kappa_{0}}{\sqrt{\varepsilon_{0}}} r\right) \\
G_{x y}^{01}(r) & =-\frac{1}{k_{\mathrm{B}} T} Q_{x}^{0}(\nabla) Q_{y}^{1}(-\nabla) \frac{1}{\varepsilon_{0} \varepsilon_{1} r} \frac{\kappa_{0}^{2}}{\varepsilon_{0}}\left(\frac{\kappa_{1}^{2}}{\varepsilon_{1}}-\frac{\kappa_{0}^{2}}{\varepsilon_{0}}\right)^{-1} \\
& \times\left[\exp \left(-\frac{\kappa_{0}}{\sqrt{\varepsilon_{0}}} r\right)-\frac{\kappa_{1}^{2} \varepsilon_{0}}{\kappa_{0}^{2} \varepsilon_{1}} \exp \left(-\frac{\kappa_{1}}{\sqrt{\varepsilon_{1}}} r\right)\right] \\
G_{x y}^{\mathrm{c}, 11}(r) & =-\frac{1}{k T} Q_{x}^{1}(\nabla) Q_{y}^{1}(-\nabla) \frac{1}{\varepsilon_{1} r} \exp \left(-\frac{\kappa_{1}}{\sqrt{\varepsilon_{1}}} r\right) \\
G_{x y}^{12}(r) & =\frac{1}{k T} Q_{x}^{1}(\nabla) Q_{y}^{1}(-\nabla) \frac{1}{\varepsilon_{0} \varepsilon_{1}^{2} r}\left(\varepsilon_{0}-1\right)\left(1-\frac{1}{2} \frac{\kappa_{1}}{\sqrt{\varepsilon_{1}}} r\right) \exp \left(-\frac{\kappa_{1}}{\sqrt{\varepsilon_{1}} r} r\right) \\
& +\frac{1}{k T} Q_{x}^{1}(\nabla) Q_{y}^{1}(-\nabla) \frac{1}{\varepsilon_{0} \varepsilon_{1}^{2} r} \frac{\kappa_{0}^{4}}{\varepsilon_{0}^{2}}\left(\frac{\kappa_{0}^{2}}{\varepsilon_{0}}-\frac{\kappa_{1}^{2}}{\varepsilon_{1}}\right) \\
& \times\left[\exp \left(-\frac{\kappa_{0}}{\sqrt{\varepsilon_{0}}} r\right)-\left(1-\left(1-\frac{\kappa_{1}^{2} \varepsilon_{0}}{\kappa_{0}^{2} \epsilon_{1}}\right) \frac{\kappa_{1}}{\sqrt{\varepsilon_{1}}} \frac{r}{2}\right) \exp \left(-\frac{\kappa_{1}}{\sqrt{\varepsilon_{1}} r} r\right)\right] .
\end{aligned}
$$

The results obtained for ion-ion screening potential coincide with the result [8] only for the case $\varepsilon_{0}=1$. In order to understand the reason of such difference we consider ionic mean force potentials at the infinite dilution of ions which play the role of interionic potentials for considering the system in the McMillan-Mayer level of a description [11]. At the infinite dilution of ions from expressions (19)-(23) we 
have

$$
\begin{aligned}
& W_{\mathrm{ab}}^{00}(r)=e^{2} z_{a}^{0} z_{b}^{0} \frac{1}{\varepsilon_{0} r}, \quad W_{\mathrm{ab}}^{01}(r)=e^{2} z_{e}^{0} z_{b}^{1} \frac{1}{\varepsilon_{0} \varepsilon_{1} r}, \\
& W_{\mathrm{ab}}^{\mathrm{c}, 11}(r)=e^{2} z_{a}^{1} z_{b}^{1} \frac{1}{\varepsilon_{1} r}, \quad W_{\mathrm{ab}}^{12}(r)=-e^{2} z_{a}^{1} z_{b}^{1} \frac{\varepsilon_{0}-1}{\varepsilon_{0} \varepsilon_{1}^{2}} \frac{1}{\sqrt{r}} .
\end{aligned}
$$

At the same time for the model used in [8]

$$
W_{\mathrm{ab}}^{00}(r)=\frac{e^{2} z_{a}^{0} z_{b}^{0}}{\varepsilon_{0} r}, \quad W_{\mathrm{ab}}^{01}=e^{2} z_{a}^{0} z_{b}^{1} \frac{1}{\varepsilon_{1} r}, \quad W_{\mathrm{ab}}^{\mathrm{c}, 11}(r)=\frac{e^{2} z_{a}^{1} z_{b}^{1}}{\varepsilon_{1} r}, \quad W_{\mathrm{ab}}^{12}(r)=0 .
$$

We can see that both models coincide only for the case $\varepsilon_{0}=1$. The principal difference of the model (23) compared to (24) is the presence of the blocked part $W_{\mathrm{ab}}^{12}(r)$ in interionic potentials which describes the interactions through the dielectric matrix. The expression (23) also corrects fluid-matrix interionic interactions. We should note that interionic interactions have got Coulomb form with effective dielectric constant

$$
\varepsilon_{\mathrm{ef}}=\varepsilon_{1} \frac{\varepsilon_{1} \varepsilon_{0}}{\varepsilon_{0}\left(\varepsilon_{1}-1\right)+1} .
$$

Since $\varepsilon_{1} \varepsilon_{0} \geqslant \varepsilon_{0}\left(\varepsilon_{1}-1\right)+1, \varepsilon_{\text {ef }} \geqslant \varepsilon_{1}$. In particular $\varepsilon_{\text {ef }}=\varepsilon_{1}$ for $\varepsilon_{0}=1$ and $\varepsilon_{\text {ef }}=\varepsilon_{0}$ for $\varepsilon_{1}=1$. Nevertheless, according to the scheme of describing the effects of quenched disorder on the fluid properties we should separate the interionic potential into connected and blocked parts

$$
W_{\mathrm{ab}}^{11}(r)=W_{\mathrm{ab}}^{\mathrm{c}, 11}(r)+W_{\mathrm{ab}}^{\mathrm{b}, 11}(r) .
$$

It is not difficult to show that the ROZ equations (2)-(5) with approximations (10) for the model (23) lead to the interionic screening potentials in the form (19)(22).

The analysis of the result (19)-(22) shows that as for the usual ion-dipole case [11-12] resulting from ionic screening, all electrostatic interactions (ion-ion, iondipole and dipole-dipole) decay exponentially. As in [8] connected and blocked parts of the fluid-fluid screening potentials have opposite signs and different asymptotics. The connected part decay as $\frac{1}{r} \exp \left(-\frac{\kappa_{\alpha}}{\sqrt{\varepsilon_{\alpha}}} r\right)$, the blocked part has more long-range asymptotic $\exp \left(-\frac{\kappa_{1}}{\sqrt{\varepsilon_{1}}} r\right)$. Hence, the screening potentials $G_{x y}^{11}(r)$ can change their signs for intermediate distances. Such interparticle ordering was observed in [8-10] for ionic systems and we see that resulting from ionic screening the similar ordering is possible for dipolar molecules as well.

\section{References}

1. Given J.A., Stell G. // J. Chem. Phys., 1992, vol. 97, p. 4573.

2. Given J.A., Stell G. // Physica A, 1994, vol. 209, p. 495.

3. Lomba E., Given J.A., Stell G., Weis J.J., Levesque D. // Phys. Rev. E, 1993, vol. 48, p. 233. 
4. Vega C., Kaminsky R.D., Monson P.A. // J. Chem. Phys., 1993, vol. 99, p. 3003.

5. Kierlik E., Rosinberg M.L., Tarjus G., Monson P.A. // J. Chem. Phys., 1997, vol. 106, p. 264.

6. Trokhymchuk A.D., Pizio O., Holovko M.F., Sokolowski S. // J. Chem. Phys., 1996, vol. 100 , p. 17004.

7. Trokhymchuk A.D., Pizio O., Holovko M.F., Sokolowski S. // J. Chem. Phys., 1997, vol. 106 , p. 220.

8. Hribar B., Pizio O., Trokhymchuk A., Vlachy V. // J. Chem. Phys., 1997, vol. 107, p. 6335 .

9. Hribar B., Pizio O., Trokhymchuk A., Vlachy V. // J. Chem. Phys., 1998, vol. 109, p. 2480.

10. Bratko D., Chakraborty A.K. // J. Chem. Phys., 1996, vol. 104, p. 7700.

11. Yukhnovsky I.R., Holovko M.F. The Statistical Theory of the Classical Equilibrium Systems. Kiev, Naukova Dumka, 1980 (in Russian).

12. Golovko M.F., Yukhnovsky I.R. In: The Chemical Physics of Solvation. Amsterdam, Elsevier, 1985, vol. A, p. 207-262.

\section{Екрановані потенціали для іонно-дипольної системи, адсорбованої на іонно-дипольних невпорядкованих матрицях}

\section{М.Ф.Головко, З.В.Поліщук}

Інститут фізики конденсованих систем НАН України, 290011 Львів, вул. Свєнціцького, 1

Отримано 4 вересня 1998 р.

Розглядається метод репліки для рівнянь Орнштейна-Церніке для іонно-молекулярної рідини, адсорбованої в невпорядкованій іонномолекулярній матриці. Отримано явні вирази для екранованих потенціалів для іонно-дипольного випадку. Показано, що флюїд-флюїд міжіонний потенціал розкладається на зв'язуючу і блоковану частини.

Ключові слова: метод репліки для рівнянь Орнштейна-Церніке, екрановані потенціали, пористе середовище, іонно-молекулярна система

PACS: $61.20,05.20$ 